\title{
Rheumatoid Arthritis with Deep Venous Thrombosis (DVT): A Case Report
}

\author{
Muhammad Shaista Ali Khan', Mohammad Saiful Habib², AK M Motiur Rahman Bhuiyan³, Md. Mizanur Rahman \\ Khan $^{2}$, Mostofa Midhat Pasha' ${ }^{2}$, Hasan Imam², Md. Rafiqul Alam ${ }^{3}$, Taimur AK Mahmud ${ }^{4}$ \\ ${ }^{1}$ FCPS (Medicine) Course Student, ${ }^{2}$ Medical Officer, ${ }^{3}$ Assistant Professor, ${ }^{4}$ Professor, Department of Medicine, Bangabandhu Sheikh Mujib \\ Medical University, Dhaka.
}

\begin{abstract}
A 25-year-old lady presented with pain and swelling with restricted movement of right lower limb for one month. She had arthritis involving multiple large and small joints of hands and feet over the last 4 years and was diagnosed as a case of rheumatoid arthritis (RA) 2 years back. She had been taking disease modifying antirheumatic drugs (DMARD) methotrexate since then and continued it for one year. Subsequent investigations revealed that she developed deep venous thrombosis (DVT) of right posterior tibial and calf veins but no other predisposing factor was found. This case emphasize on the consideration of developing DVT in patients with Rheumatoid arthritis.
\end{abstract}

[BSMMU J 2010; 3(2): 10-11]

\section{Introduction:}

Rheumatoid arthritis (RA) is a common, chronic, and systemic inflammatory autoimmune disease. Although RA preferentially targets the synovial lining of the joints, it can affect other organ sys-tems including the lungs, heart, and blood vessels. ${ }^{1}$ Extraarticular manifestations of the systemic disease can occur in up to $46 \%$ of patients with RA over a 30-year period from disease onset. ${ }^{2}$

Rheumatoid arthritis is not generally considered to be a risk factor for venous thromboembolism (VTE), although abnormalities of coagulation factors have been found in patients with rheumatoid arthritis. ${ }^{3}$ The excess risk of noncardiac vascular disease in RA is likely to be related, in part, to the systemic inflammation associated with the extraarticular manifestations of RA. ${ }^{2}$

\section{Case Report:}

A 25-year-old housewife of middle class family hailing from Manikgonj got admitted in BSMMU Hospital on January21, 2010 with the complaints of severe pain and swelling with restricted movement of right lower limb for one month. She had been suffering from pain and swelling of multiple small and large joints of hands and feet e.g proximal interphalangial joints (PIPs), metacarpophalangial joints (MCPs), wrist joints, knee joints bilaterally with morning stiffness $(>1 \mathrm{hr}$ ) over the last 4 years and was diagnosed as a case of Rheumatoid arthritis (RA ) 2 years back on basis of clinical background and positive rheumatoid factor (RF). Since then she had been taking methotrexate (10 mg weekly) and continued it for 1 year

Address for corrodpondence: Dr. Muhammad Shaista Ali Khan, Department of Medicine, Room No: 624, Block - C, BSMMU, Shahbag, Dhaka-1000, Bangladesh. Email: taimur@bsmmu.org and took analgesic (indomethacin) occasionally. She has no history of rash, hair loss, and oral ulceration, redness of eyes, back pain or fetal loss. She had been normally menstruating, did not take any contraceptive and had no significant past medical, surgical or obstetrical history and she has no history of prolong immobilization.

On general examination, patient had average body built with BMI $18.6 \mathrm{~kg} / \mathrm{m}^{2}$, mildly anaemic, non icteric, pulse 84 beats/min, BP - 110/75 mm of Hg, no dependant oedema or lymphadenopathy. Musculoskeletal system examination reveals right leg swollen upto the knee; red, warm and with pitting oedema. The peripheral pulses were normal and the superficial veins were not prominent. Both active and passive movements of knee and ankle joints of right leg were restricted. Also, there was muscle wasting with dorsal guttering, swelling of $3^{\text {rd }}$ and $4^{\text {th }}$ PIPs and ulnar deviation in both hands. Other systems revealed nothing abnormal.

Laboratory investigation showed Hb-10.8 gm/dl, ESR-62 $\mathrm{mm}$ in $1^{\text {st }}$ hour, WBC- $14 \times 10^{9} / \mathrm{L}$, Platelets-380 $\times 10^{9} / \mathrm{L}$, PBFdimorphic blood picture, Serum alanine transaminase (ALT)-64 u/L, S. uric acid - 4.1mg/dl. Both antinuclear antibody (ANA) and anticardiolipin antibody tests were negative. Duplex examination of right lower limb vessels reveals deep venous thrombosis (DVT) of right posterior tibial and calf veins along with chronic venous insufficiency (CVI) of right lower limb. Patient was treated with low molecular weight heparin and warfarin and was discharged after clinical improvement and target INR report.

\section{Discussion}

Rheumatoid arthritis (RA) is a chronic systemic inflammatory disease of unknown etiology that primarily 
targets synovial tissues. It is relatively common with a prevalence of approximately $1 \%$ in adults all over the world. ${ }^{4}$

There are various extra-articular manifestations of rheumatoid arthritis that are associated with increased morbidity and mortality. Study shows extra-articular manifestations can occur in up to $40 \%$ of patients with RA over a 30 -year period from disease onset. ${ }^{5}$ Deep vein thrombosis (DVT) till to-date is not generally considered to be among those of extra-articular manifestations of RA. However, data strongly suggesting rheumatoid arthritis to be a risk factor for developing venous thromboembolism (VTE) esp. DVT in hospitalized medical patients.

To determine the incidence of venous thrombo-embolism (VTE) in hospitalized patients with rheumatoid arthritis, a study conducted in USA with data obtained from National Hospital Discharge Survey (NHDS). The number of patients discharged from non-Federal short stay hospitals throughout the United States from 1979 to 2005 with a discharge code for rheumatoid arthritis showing DVT was diagnosed in 79,000 of 4,818,000 (1.64\%) patients with rheumatoid arthritis and no joint operation. ${ }^{3}$

A community based cohort study consisted of 609 patients with rheumatoid arthritis diagnosed between January 1, 1955 and December 31, 1994 was carried out in Olmsted County, Minnesota with median follow up of 11.8 years. The 30-year cumulative incidence of venous thromboembolic events comprising DVT and pulmonary embolism was estimated to be $7.2 \%{ }^{2}$

In this reported case, all other possible causes that might contribute to development of DVT have been carefully excluded. cox 2 inhabitors are sometimes associated with thromboembolic episodes; however, our patient was taking indomethacin (cox 1 inhibitor) as and when necessary. ${ }^{6}$ So the cause of DVT in this patient might be Rheumatoid arthritis.

\section{Conclusion}

Thus, review of various medical literatures provides data suggesting rheumatoid arthritis to be a risk factor for venous thrombo-embolism (VTE) in hospitalized medical patients. So, the relationship between rheumatoid arthritis and DVT or PE should be further studied and heightened awareness of the risks of venous thrombo-embolism would be appropriate in caring for hospitalized patients with rheumatoid arthritis.

\section{References:}

1. Angelo G, Kenneth G, Jeffrey R. Treatment of rheumatoid arthritis. Am J Health Syst Pharm 2006;63:2451-65.

2. Kimberly PL, Kelly VL, Eric LM, Robyn LM, Teresa JH, Carl T. Incidence of Noncardiac Vascular Disease in Rheumatoid Arthritis and Relationship to Extraarticular Disease Manifestations. Arthritis Rheum 2006;54:642-48.

3. Fadi M, Ravinder S, Abdo Y, Yaekoub1, Reiad N, Paul D, et al. Risk of venous thromboembolism with rheumatoid arthritis. Thrombosis and Haemostasis 2009;101(1):134-138.

4. Lee Goldman, Dennis A. Ausiello, eds. Cecil Textbook of Medicine. 23 ${ }^{\text {rd }}$ edn. Philadelphia: WB Saunders; 2009.1644p.

5. Turesson C, O'Fallon WM, Crowson CS, Gabriel SE, Matteson EL. Extra-articular disease manifestations in rheumatoid arthritis: incidence trends and risk factors over 46 years. Ann Rheum Dis 2003;62:722-7.

6. Konstam MA , Weir MR , Reicin A. Cardiovascular thrombotic events in controlled, clinical trials of rofecoxib. Circulation 2001;104:2280-2288. 